\title{
The Case for Occupant-Centric Daylight Analytics: a Comparison of Horizontal Illumination and Immersive View
}

\author{
Siobhan Rockcastle ${ }^{1,2}$, María L. Ámundadóttir ${ }^{3}$, Marilyne Andersen ${ }^{2}$ \\ ${ }^{1}$ Baker Lighting Lab, University of Oregon, Eugene, United States \\ ${ }^{2}$ LIPID Lab, École polytechnique fédérale de Lausanne, Lausanne, Switzerland \\ ${ }^{3}$ OCULIGHT Analytics, Reykjavik, Iceland
}

\begin{abstract}
Daylight is valued as an energy efficient alternative to electric light and over the past few decades, climate-based horizontal illuminance metrics have demonstrated the importance of variable sky conditions on annual predictions. These metrics allow us to evaluate a building as an ensemble of spaces that can achieve a pre-set performance target. In addition to daylight's ability to offset electric lighting, it can also impact psychological and physiological responses in building occupants, such as discomfort from glare, alertness, mood or sleep quality through non-visual effects, and emotion from perceived contrast and composition, all of which depend on a specific field-of-view. Occupant-centric or 'view-based' models pertaining to daylight indoors can provide complementary point-in-time or aggregated predictions of an occupant's response over time, but are difficult to compare to horizontal illuminance or 'task-based' metrics in a simulation environment, because the sensor position, view direction, and time granularity vary greatly between each approach. This paper uses a simulated office floor plate with varied seating and wall configurations to propose a method of scoring task-based and view-based metrics, allowing for a relative comparison. The results of this comparison reveal a disconnect in performance narratives between each approach, supporting the need for further work to develop an integrated approach for comparing building and occupant-scale performance considerations.
\end{abstract}

\section{Introduction}

Over the last several decades, daylight has been valued as an energy efficient alternative to electric light. Architects are taught to integrate daylight while avoiding the risk of visual discomfort and overheating by integrating appropriate shading and control strategies. This valuation of daylight has positioned the building as an object that can perform, using illuminance thresholds and energy consumption as quantities to optimize and human discomfort as a risk to mitigate. The emergence of climate-based daylight metrics and their integration into standards aimed at improving the integration of daylight in buildings has encouraged both architects and consultants to acknowledge the impact of climate dynamics on daylight performance predictions. Metrics like Useful Daylight Illuminance (UDI), Daylight Autonomy (DA) or, more recently, Spatial Daylight Autonomy (sDA) and Annual Sunlight Exposure (ASE), have emerged with increased consensus from academic and industry partners, and the latter are currently being adopted into standards like IES LM-83 (IES, 2014). Despite this slow and steady shift from static illuminance metrics toward more dynamic and realistic simulation protocols using climate-based metrics, horizontal task illumination is only one part of a rather complex web of human needs associated with daylight and does not evaluate a handful of other very important factors that concern human physiology, behavior, and perception (Galasiu \& Veitch, 2006). Our understanding of these impacts has shifted in large part due to emerging research in photobiology and neuroscience, but our ability to evaluate these impacts in the building design process alongside existing considerations is still quite limited. There are a number of reasons for these limitations, some practical and others reinforced through the existing analytical methods we have come to rely on.

\section{Task-based metrics}

Early daylight performance metrics, such as Daylight Factor (DF) (Moon \& Spencer, 1942), emerged as a response to property right disputes, where the right to light in rapidly densifying cities demanded a means to measure the relative access of diffuse sky light indoors. In the pursuit of energy-efficient daylight integration over the past few decades, climate-based metrics like DA, UDI, sDA, and ASE emerged in response to the notion that sun, climate, and occupant dynamics made simplified metrics such as DF or point-in-time illuminance unreliable predictors of actual daylight penetration (Mardaljevic \& Heschong, 2009).

Whether static or climate-based, these task-based metrics all use a similar approach to evaluating a space or a building: a grid of task-level illuminance sensors is generated across a floor area and performance is achieved if a desired percentage or a mean value of that occupied area meets a defined instantaneous or annual target. This mode of valuation positions the floor area as a territory 
and the building envelope as a boundary that allows that territory to perform.

\section{View-based metrics}

View-based metrics are evaluated using sensors positioned at the eye-level to capture the occupant experience in space. Daylight Glare Probability (DGP) is broadly accepted in the daylight community as a robust method for predicting glare risk from daylight. DGP is commonly calculated for individual seating positions, assuming either a fixed view-direction (Weinold, 2011) or an adaptive zone (Jakubiec \& Reinhart, 2011). Visual comfort metrics (DGP and others that preceded it) were transformative in the sense that they evaluated the performance of an occupant response instead of the taskbased capacity of a space. While illuminance metrics are based on thresholds that are deemed adequate for task activities to be comfortably achieved and are thus inherently 'occupant-centric,' the propagation of those values to a grid of points across an occupied floor area removes the specific occupant as the performance target and replaces it with a territory of space where possible visual tasks could be adequately performed.

Over the past decade, research on the non-visual effects of light has brought more attention to the importance of light exposure in people's well-being. The consequences of not achieving adequate light exposure have been linked to a broad range of health-related conditions as well as alertness and sleep quality. Knowledge about the nonimage-forming effects of light have for instance been integrated into the Circadian Stimulus (CS) metric, which weights the spectral sensitivity of irradiance at the cornea and rates that spectral effectiveness using a threshold from an activating level $(\mathrm{CS}=0.1)$ to a saturation level ( $\mathrm{CS}=0.7$ ) (Rea \& Figueiro, 2016). CS opened the door for applied measures in the area of non-visual health, but it was developed from the acute melatonin suppression levels following a one hour light exposure. In addition to the limitation that melatonin naturally peaks during the night, the CS metric does not integrate exposure levels over time nor does it account for prior light history, which are critical factors when predicting the physiological effect of (day)light over time. Timing of light exposure affects our system in a complex and cumulative way, where fluctuations over the day due to solar geometry, sky condition, and time of year are likely to induce non-linear responses (Ámundadóttir, 2016).

The non-visual Direct Response (nvRD) model goes beyond instantaneous exposure and predicts daily light dose based on cumulative vertical illuminance accounting for the ipRGC spectral effectiveness (Ámundadóttir, 2016). The daily nvRD light dose predicts the potential of accumulated light exposure to impact the feelings of vitality and alertness accounting for both spectral and temporal changes in light exposure. Results are rated using thresholds that correspond to the number of 'vital' hours in a day, where the recommended daily dose is achieved after 8.4 hours of effective light exposure (Amundadottir et al., 2017).

The emotional impacts of daylight have also been integrated into new prediction models such as modified Spatial Contrast (mSC), which proposes thresholds for determining the impact of daylight composition on ratings of calm or excitement (Rockcastle et al., 2017). This model was developed from experimental data on subjective ratings of rendered daylit scenes. While emotion is composed of multiple dimensions and $\mathrm{mSC}$ only accounts for ratings of calming to exciting, it opens the door for other algorithms that can relate daylight distribution to other emotional responses in architecture.

\section{Simulation-based daylighting approaches}

There are a number of software tools used to compute task-based metrics like sDA and ASE as well as glarebased metrics like DGP. DIVA-for-Rhino, LightStanza and Sefaira have risen in popularity over the last several years due in large part to their development of intuitive graphic user interfaces. LightStanza gives users the ability to run cloud-based simulations and compare the performance of design alternatives through a score card approach. These tools integrate task-based metrics alongside DGP, but they do not integrate computational models for occupant-centric performance in novel-areas like non-visual health and visual perception.

Simulation-based tools that integrate CS and melanopic lux have emerged in recent years to calculate the instantaneous non-visual effects of light (Konis, 2016) (Solemma, 2018) where Leslie (2011) was the first to introduce CS as a part of integrated daylighting approach. To analyze the cumulative and dynamic effects of light on an occupant's field-of-view, the nvRD metric was recently integrated into a workflow alongside DGP and $\mathrm{mSC}$ to offer an occupant-centric approach to daylight performance, where eye-level predictions were computed for a range of view directions from a fixed view position over time (Amundadottir, Rockcastle, Sarey Khanie, \& Andersen, 2017). Building on this work, Rockcastle et al, $2017 \mathrm{~b}$ offered a multi-position approach which accelerated the workflow from an individual occupant to a building-scale analysis, allowing daylight performance to be computed across multiple skies, time steps, view position and view directions. Furthermore, Rockcastle et al. 2018 introduced a web-based visualizer called OCUVIS that allows for the interactive exploration of data, including the performance of illuminance metrics like DA, ASE, and DF, using floor and program tags to 
query and aggregate results. While this approach allows the user direct interaction with a broad array of performance metrics, annual dynamics, and spatial view positions, there is no clear method to compare the relative performance of view-based metrics like nvRD, DGP, and mSC to task-based metrics like DF, UDI, DA or ASE.

Task-based metrics were designed to assess an array of sensor points across an occupied floor area and determine a percentage of those sensors that achieve a given target. View-based metrics were designed to assess a single and defined view position and have been applied to an array of view positions and directions without a set of rules or recommendation for how many or how far apart these positions should be within an occupied floor area. When they are applied, task-based metrics compute performance across a densely-spaced sensor grid, and view-based metrics compute the performance of aggregated occupant view positions. The time granularity (instantaneous or daily vs. annual), sky (clear vs. overcast vs. stochastic), and sensor placement (eye-level immersive vs. horizontal grid) vary between task-based and view-based methods, making them difficult to compare.

\section{Simulation framework}

To compare the daylight performance of task-based and view-based metrics, simulations were run for a series of small office layouts and orientations using a new aggregate scoring method. Based on this method, viewbased performance scores (which account for nvRD, mSC, and DGP) are compared to task-based scores (DA and ASE) in order to discuss the impact of layout, orientation, and seating position on occupant performance.

This study considers a generic side-lit office with 3 layout types: 'private', team' and 'open' (Figure 1). The offices are located in Portland, OR (45.5 N, 122.6 W). All three types include both North and South facing offices and a range of seating configurations that face both East and West., resulting in 7 unique layout conditions:

- The 'open' layout is a single $84 \mathrm{~m}^{2}$ open office with 10-20 people per office.

- The 'team' layout is a $38 \mathrm{~m}^{2}$ space with 4-8 people per office (North and South facing).

- The 'private' layout consists of 4 partitioned office spaces of $18 \mathrm{~m}^{2}$ each with single or double occupancy.

Figure 1a)-c) shows a selection of these occupancies for each layout; private, team, and open.

Sensor nodes for task-based metrics were simulated at desk height (28 inches from the floor) with an 18 inch spacing between nodes and 12 inch-offset from adjacent walls (Figure 1a-c).

As furniture and seating arrangement impacts direct and reflected photometric quantities used to compute metrics like mSC, DGP, and nvRD, simulated view positions should ideally be positioned where an occupant is likely to sit or stand for long periods of time, and models should include furniture and other visually intrusive objects.

View positions for occupant-centric metrics were set at seated eye-level (42 inches from floor) and were simulated facing either due East or due West with $\pm 45^{\circ}$ and $\pm 90^{\circ}$ rotational freedom (i.e. 5 view directions per occupant) as seen in Figure 1d). The simulated seating locations for each occupant are shown in Figure 1a)-c).
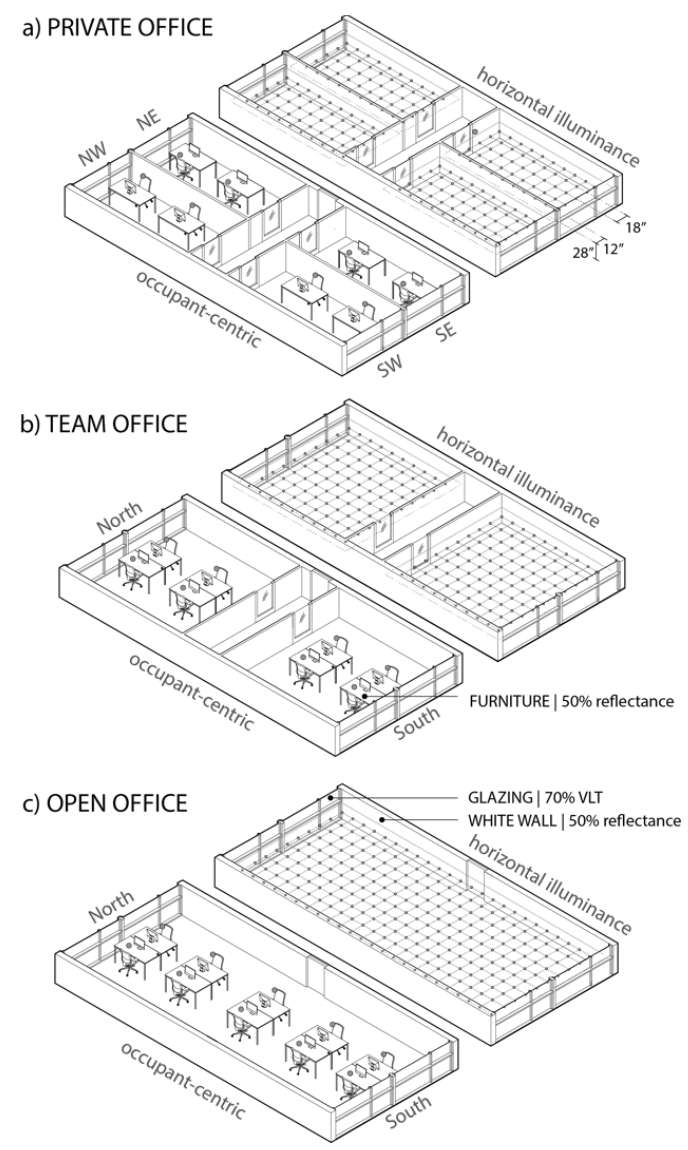

d) VIEW DIRECTIONS

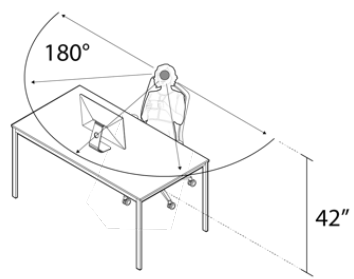

Figure 1: Space configurations: a) private, b) team, and c) open., with d) Showing the 5 view dir. for each position facing $E$ or $W$ with $\pm 45^{\circ}$ and $\pm 90^{\circ}$ rotation.

To compute horizontal task-based metrics (DF, DA, ASE), DIVA-for-Rhino was used to run RADIANCE and DAYSIM. Climate-based metrics were simulated using the Perez sky model (gendaylit). RADIANCE materials were then set to default reflectance or 
transmittance values for floor (0.40), walls $(0.70)$, ceiling (0.80), mullions $(0.50)$, glazing $(0.70)$, furniture (0.50), and computer ( 0.70 for casing, 0.20 for glass in off mode). The spaces were simulated without external blinds.

As 3-phase or 5-phase annual simulations would be very computationally expensive for view-based metrics, the authors propose to use the "Lightsolve" approach instead. This method subdivides the year into 56 annual moments (7 monthly and 8 daily) and categorizes the sky into four profiles: clear, clear turbid, intermediate, and overcast (Kleindienst et al, 2008 and Kleindienst \& Andersen, 2012). Climate-based results for mSC, DGP, and nvRD use a weighted combination of those four sky profiles based on the frequency of each sky condition in the weather file. Using the Perez sky model to simulate the relevant sky conditions, this approximate 'climate-based' method of aggregating view-based values into a single annual value is a first attempt at generating a synthetic measure that can be compared to compact climate-based metrics like DA and ASE.

All the renderings used as inputs for view-based metrics (mSC, DGP, and nvRD) were generated using RADIANCE. The workflow used in this paper builds on the method proposed in Rockcastle et al., 2017 but evolves from CIE clear and overcast sky models to a climate-based approach using the Perez sky model.

The authors acknowledge that further development is still needed to refine this approach and the results presented are meant to serve as a proof-of-concept that can motivate future work.

\section{Scoring and ranking method}

To produce an aggregate score for task-based and viewbased performance metrics, we developed a scoring method to rank the different office configurations simulated for this paper. This allows us to compare task and view-based analyses in relative terms.

\section{Multi-criteria scoring method}

Multi-criteria scoring is not a novel approach in building performance evaluation. WELL v2 operates on a pointbased system, with a total of 110 points available to each project (https://v2.wellcertified.com/v/en/overview). All optimizations have maximum point-values, with the point-value of each feature determined by its potential for impact. This is defined as the extent to which a feature addresses a specific health and wellness concern or opportunity for health promotion, and the potential impact of effective intervention.

All parts in optimizations hold a point-value equal to or less than the optimization maximum. Projects may pursue parts under the optimization to accrue points up to the maximum point-value established for the optimization. WELL v2 offers 3 points each for circadian lighting, glare control, and enhanced daylight and while this equal weighting is not based in rigorous field-testing, the composite score offers a holistic multi-criteria approach.

The scoring approach in this paper uses target levels based on recommended thresholds from IES LM-83. For each metric, the authors defined a threshold value across daylit hours and under climate-based sky conditions in order to calculate a composite score.

The task-based score was calculated using DA and ASE. DA was evaluated using target work plane illuminance of 3001x, 5001x, and 7001x; since the space is used as an office space. A score of 2 is assigned if $75 \%$ of space exceeds a given illuminance threshold ( $300 \mathrm{~lx}, 500 \mathrm{~lx}, 700$ lx) for at least $50 \%$ of occupancy hours. Only a score of 1 is assigned if the threshold exceeds $55 \%$ of the space. This approach gives a total maximum score of 6 , with a higher score indicating a brighter space. To account for the potential of overlit area due to direct solar exposure, the percentage of space with ASE (1000 lx, +250 hours) above $10 \%$ of the floor area was used to apply a negative score of 2 .

To score the view-based results, a percentage of view directions was used instead of floor area as a relative analogy. The DGP and $\mathrm{mSC}$ values for each instance were rated using the thresholds ( 0.40 for DGP and 7 for $\mathrm{mSC}$ ) and then counted as the percent of sensors that either satisfy this threshold or not. The same was done for nvRD except on a daily basis and was then made into a weighted sum according to sky type occurrence for climate-based comparison. This allows us to offer an occupant-centric evaluation for annual climate-based performance.

To determine targets for this percentage, the IES LM-83 standard as well as the annual DGP approach (Weinold, 2011) were used as references. A score of 2 was assigned if $75 \%$ or more view directions surpassed a given threshold and a score of 1 was assigned for $55 \%$ of view directions. To account for the risk of glare, at most $5 \%$ of view directions should exceed the glare-risk threshold to get a performance score, in that case a score of 2 was subtracted from the total score. Each metric (nvRD, mSC, DGP) contributes a maximum of 2 to the total score. Thus the maximum score is 6 and offers a possible implementation of occupant-centric annual performance that can be compared to task-based illuminance metrics.

The authors acknowledge that the selected thresholds of $55 \%$ and $75 \%$ (of all simulated view directions) may be considered arbitrary and while they are based on values implemented in IES LM-83's application of sDA (percent of usable floor area reaching a target daylight 
performance), further research would be needed to determine if they are appropriate for view-based metrics. These thresholds should therefore be considered as proofof-concept and are only useful in their capacity to compare performance between methods in relative terms.

\section{Ranking as a visual representation}

To compare the rate of change and rank-order of different space configurations using the developed scoring method we have chosen a rank diagram. Each set of results are ordered according to the computed score and then compared by connecting each group's values on one type of results to their values on the second type of results. What this visualization aims to reveal is whether each group of office configurations increases, decreases, or remains similar between human-centric and horizontal illuminance results.

\section{Results}

Results have been organized into two sections: climatebased summary and impact of view directions. All results were run without shading systems to allow for a direct comparison between task-based and view-based metrics as there currently is no control algorithm designed to trigger an adaptive shading system for emotion metrics (nor for non-visual effects, although their cumulative nature would probably make them a trigger for shade reopening).

\section{Climate-based results}

Figure 2 shows tables including the percentage of view directions and floor area for occupant-centric and horizontal illuminance results, respectively, under climate-based conditions. The results are listed for each office configuration and selected performance metrics. As the authors were interested in the comparison of viewbased performance between a single view direction and a 180-degree array of view directions, we included separate view-based scores for 5 view-directions (upper section of table in Figure 2A) and 1 view-direction (lower section of table in Figure 2A). The table for horizontal illuminance in Figure 2B) contains fewer lines for the 'Private' office than in Figure 2A) as the results for task-based metrics are not influenced by whether the occupants face towards East or West.

The target thresholds used in this paper are explained below the tables in Figure 2 (for more details see previous section 'Scoring and ranking method'). The cells in the results tables are highlighted to indicate if a target level was reached or not. Light grey cells indicate that a value of 1 is contributed to the total score, while dark grey cells give a score of 2 . Unshaded values indicate that a threshold was NOT achieved and is therefore under target.
A) Occupant-centric results

\begin{tabular}{|c|c|c|c|c|c|c|}
\hline \multirow[b]{2}{*}{5 view dir. } & \multicolumn{2}{|c|}{ VITALITY } & \multirow{2}{*}{$\begin{array}{c}\text { EMOTION } \\
\mathrm{mSC}<7\end{array}$} & \multicolumn{2}{|c|}{ COMFORT } & \multirow[b]{2}{*}{ Score } \\
\hline & nvRD $\geq 4.2$ & $\mathrm{nvRD} \geq 8.4$ & & $\begin{array}{l}E v \geq 1501 x, \\
D G P<0.4\end{array}$ & $D G P \geq 0.4$ & \\
\hline Open & $85 \%$ & $51 \%$ & $69 \%$ & $72 \%$ & $9 \%$ & 1 \\
\hline Team N & $87 \%$ & $48 \%$ & $57 \%$ & $78 \%$ & $4 \%$ & 4 \\
\hline Team S & $87 \%$ & $56 \%$ & $59 \%$ & $63 \%$ & $21 \%$ & 2 \\
\hline Private NE & $85 \%$ & $45 \%$ & $90 \%$ & $75 \%$ & $3 \%$ & 5 \\
\hline Private NW & $84 \%$ & $44 \%$ & $88 \%$ & $74 \%$ & $3 \%$ & 4 \\
\hline Private SE & $85 \%$ & $54 \%$ & $84 \%$ & $64 \%$ & $17 \%$ & 2 \\
\hline Private SW & $85 \%$ & $53 \%$ & $81 \%$ & $63 \%$ & $18 \%$ & 2 \\
\hline \multicolumn{7}{|l|}{1 view dir. } \\
\hline Open & $85 \%$ & $50 \%$ & $77 \%$ & $70 \%$ & $10 \%$ & 2 \\
\hline Team N & $84 \%$ & $42 \%$ & $95 \%$ & $76 \%$ & $1 \%$ & 5 \\
\hline Team S & $93 \%$ & $60 \%$ & $51 \%$ & $63 \%$ & $26 \%$ & 1 \\
\hline Private NE & $83 \%$ & $45 \%$ & $100 \%$ & $78 \%$ & $0 \%$ & 5 \\
\hline Private NW & $71 \%$ & $26 \%$ & $100 \%$ & $63 \%$ & $0 \%$ & 3 \\
\hline Private SE & $83 \%$ & $56 \%$ & $98 \%$ & $68 \%$ & $13 \%$ & 3 \\
\hline Private SW & $96 \%$ & $63 \%$ & $65 \%$ & $61 \%$ & $30 \%$ & 2 \\
\hline
\end{tabular}

VIEW-BASED SCORE

VITALITY: $\%$ of view directions with nvRD $\geq 4.2$ larger than $\%$ of view directions with nvRD $\geq 8.4$ larger than

EMOTION: \% of view directions with $\mathrm{mSC}<7$ larger than

COMFORT: \% of view directions with Ev $\geq 1501 x$ and DGP $<0.4$ larger than

$\%$ of view directions with DGP $\geq 0.4$ larger than

\begin{tabular}{cc} 
Target & Score \\
$75 \%$ & 1 \\
$55 \%$ & 1 \\
$55 \%$ & 1 \\
$75 \%$ & 1 \\
& \\
$55 \%$ & 1 \\
$75 \%$ & 1 \\
$5 \%$ & -2 \\
& \\
\hline Max total & 6 \\
\hline
\end{tabular}

B) Horizontal illuminance results

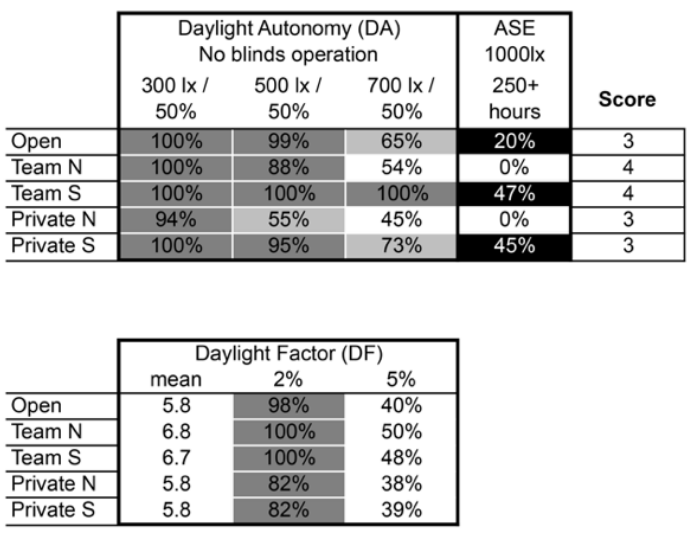

TASK-BASED SCORE

DAYLIT AREA: \% of space with DA above a certain threshold at least $50 \%$ of occupancy hours larger than

OVERLIT AREA: \% of space that is overlit should be smaller than

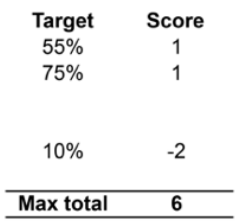

Figure 2: Climate-based summary of the multi-criteria scoring method to allow for the comparison of performance between A) occupant-centric results (view-based metrics) and B) horizontal illuminance results (task-based metrics). Results for daylight factor are also shown, but are not integrated into the task-based score. 
To indicate "bad" performance or a negative contribution to the total score, the cells are colored black. The total score is summed in the rightmost column of each table and used to rank the results plotted in Figures 3 and 4.

Figures 3 and 4 show the task-based and view-based scores for each simulated office type and orientation, based on "iterations" of metric types and based on different sky types. The produced diagrams very quickly indicate that it would be difficult to build a straightforward narrative about performance, as task- and view-based scores do vary, and sometimes greatly, for each space and orientation, while 3 out of 7 office achieve a view-based score $>3$ based on 5 view directions, only 2 out of 7 achieved a view-based score $>3$ based on a single view direction.

As is often the case in building performance simulation for dynamic factors like daylight, it is always difficult to know which metrics to prioritize and what methods deliver the most robust and holistic valuation, but acknowledging this complexity is already an important step towards a more holistic approach to daylight performance.

Analyzing Figure 3, we can see that the 'Team S' office performs well in the task-based score, but poorly for view-based scores, with performance dropping from 2 when we consider five view-directions, to only 1 when we consider a single view-direction. Alternatively, the 'Private NE' offices achieve a moderate task-based score of 3 and strong view-based scores of 5 .

Figure 4 shows a further break-down of view-based results for each office and orientation under clear, clear turbid, intermediate, and overcast skies, as well as the synthesized results for a statistical climate-based sky. Results in this figure are shown for both a single view position as well as for a percentage of 5 view-directions. The results indicate that both sky and number of viewdirections have a measurable impact on view-based performance scores. More work is needed to determine the implications of our climate-driven view-based score, as we do not know how comfort, emotion, and/or health are actually impacted under long-term exposure to a specific sky condition or series of sky conditions.

A climate-based prediction can be very useful for taskbased methods where performance may indicate an impact on energy consumption and when hourly and daily extremes may cancel one another out. On the other hand, extremes driven by sky condition may greatly impact occupant-centric responses, where glare and emotion are directly linked to contrast and sky type. For this reason, the authors are wary of presenting a climate-based prediction without also considering the clear and overcast conditions for these metrics.

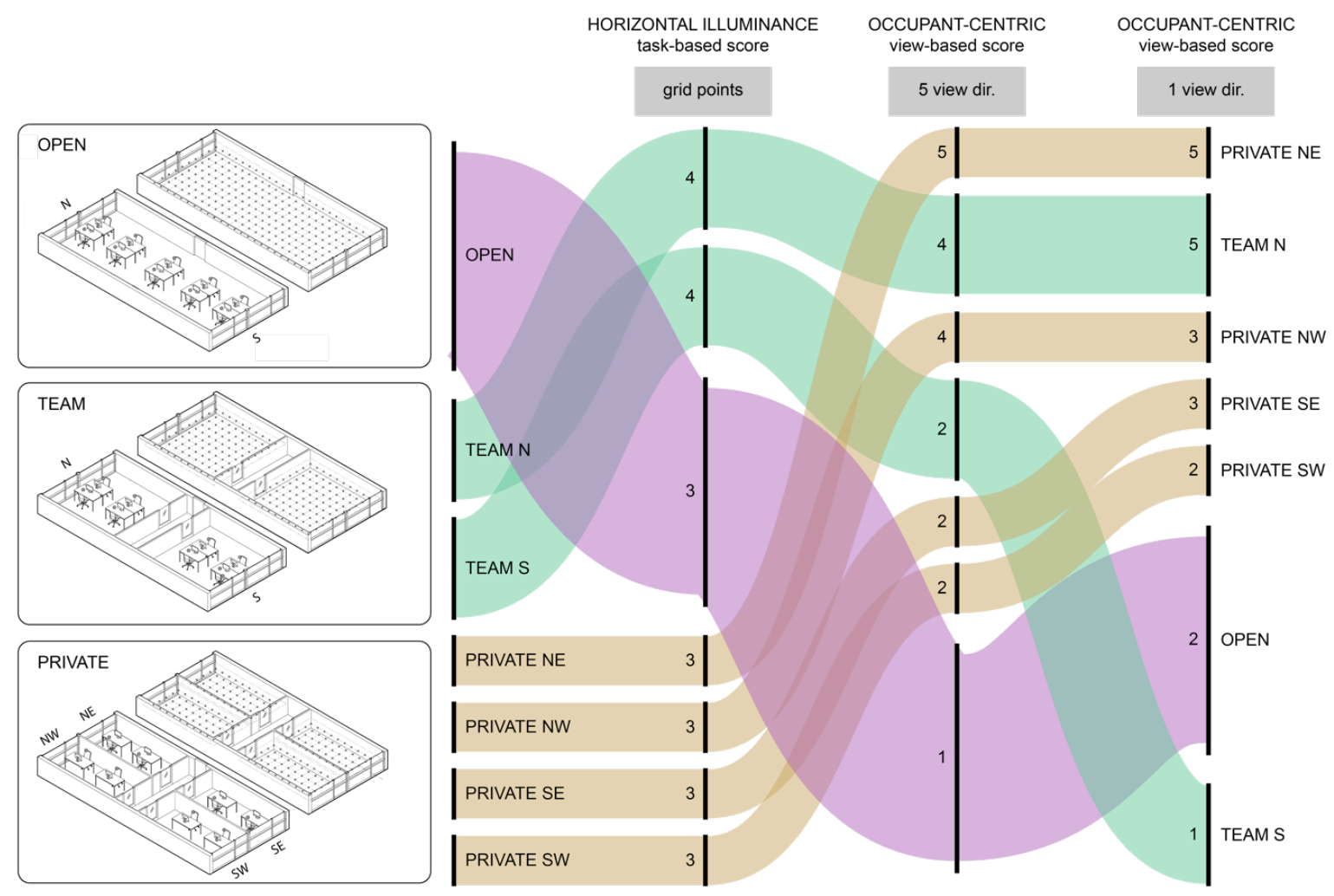

Figure 3: Diagram showing ranking of offices using a composite performance scores for horizontal illuminance metrics on the left and occupant-centric metrics on the right. The size of each category is scaled with the number of occupants in each space: open (10 people), team (4 people per office), and private (2 people per office). 


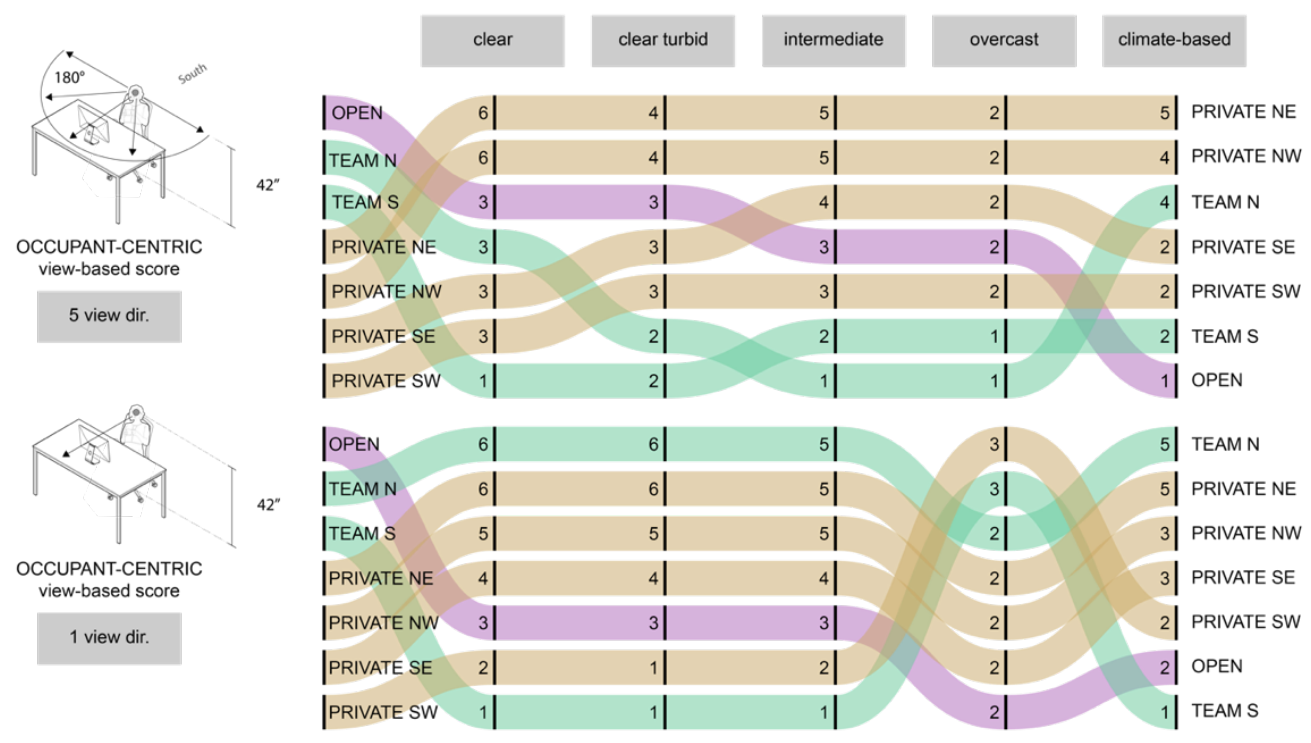

Figure 4: Diagrams showing the ranking of offices using a composite performance score for occupant-centric metrics.

\section{Impact of view direction}

While both task-based and view-based scores account for excessive daylight through either DGP or ASE metrics, task-based metrics do not account for where occupants are seating within a room and can therefore under or overestimate the risk for glare. Seating position and view orientation also have an impact on health (nvRD) and emotion (mSC), as distance from the window and daylight composition in the field-of-view will result in variable performance outcomes between occupants. At the same time, any evaluation of a building through a view-based score would be subject to seating arrangement.

As seen in Figure 3 and 4, the number of view directions analyzed per occupant can impact results. Figure 4 shows the view-based score using a single view direction compared to a more rotational freedom (5 view directions) for the simulated sky types. The difference can mainly be explained by $\mathrm{mSC}$ and DGP, which both depend on the lighting distribution in the space. Note that the light comes from the side, i.e. no occupant faces the window.

In general, 1 view direction achieves higher scores, as results are not calculated from 5 view directions (with some looking away from the window). Figure 5 refines this analysis by showing the impact of office and view orientation on individual view-based metrics for the open, team, and private office types. In these results, office type (Open, Team and Private), room orientation (North, South or Center), and view direction (East or West) impact the percentage frequency of view directions that achieve a performance threshold for each view-based metric.

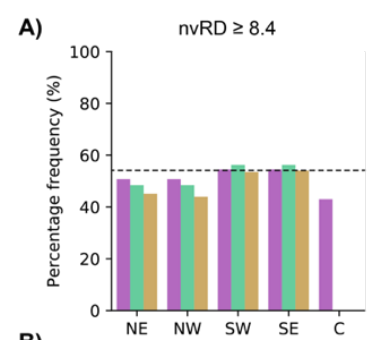

B)

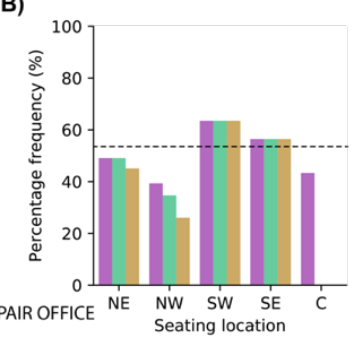

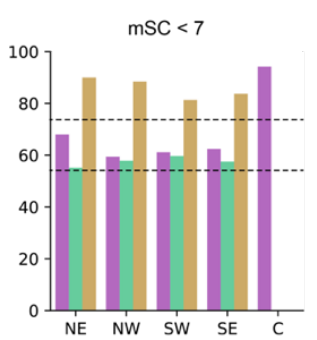

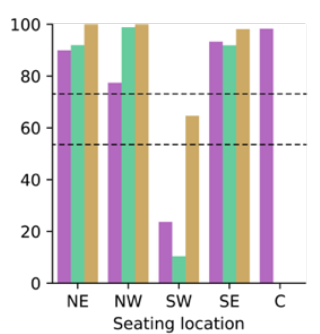

C)
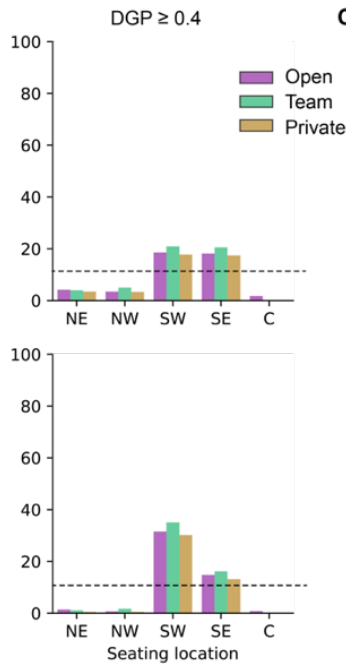

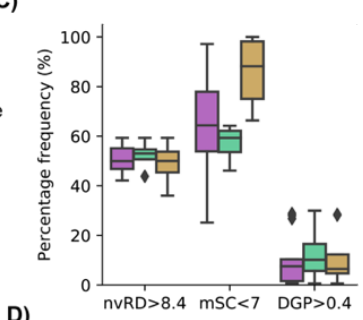

D)

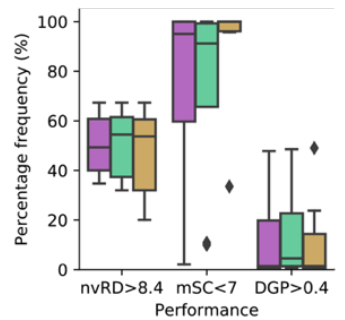

Figure 5: Percentage frequency of view directions achieving occupant-centric performance using climate-based weighting by seat locations for A) 5 view directions, and B) 1 view direction. The box plots show the distribution between different performance criteria grouped by office layout for C) 5 view directions and D) 1 view direction. 
The South-facing offices have a higher frequency of view directions above the frequency threshold for nvRD $>=8.4$ (both $\mathrm{E}$ and $\mathrm{W}$ view directions and all types). In contrast, the NW-facing private office achieves the worst performance for this metric.

East and West view directions have less of an effect on mSC values, which are more impacted by office size, with the private offices resulting in a higher frequency of view directions under the desired threshold. This may be due to the reduced percentage of views exposed to the façade, resulting in more diffuse light deeper into the space.

\section{Conclusion}

This paper reveals divergent daylight performance narratives between task-based and view-based metrics, opening a dialog about how view-based metrics may be considered alongside task-based metrics for a more holistic evaluation. The analysis presented in this paper shows that occupant-centric performance is impacted not only by office layout and orientation, but also by seating location and view direction, which can worsen an occupant's risk for glare or increase their non-visual exposure to healthy daylight during key times. While a single office layout and orientation could produce several different view-based scores depending on seating location, task-based scores rely on space rather than occupancy to determine performance. This opens up an important conversation about how we should be evaluating daylight performance for human factors at the building scale. If seating positions can produce vastly different performance outcomes in terms of comfort, health, and emotion, then perhaps a more occupantcentric approach could help us design better indoor environments for human well-being.

In addition to the larger questions brought about by this analysis, this paper also reveals the need for new algorithms that can drive dynamic shading systems based on view-based factors. While some studies have used DGP as a trigger for dynamic shading systems (Correa, da Silva, \& Andersen, 2012) there are no controllers currently on the market that can control exterior shading devices based on occupant and view-specific comfort models. It is therefore not currently possible to compare the results of metrics like sDA as defined by the LM- 83 standard (which requires shading) to the view-based metrics presented here. The authors would be intrigued to see a view-based controller that could control exterior shading systems based on models for comfort, health, or emotion.

\section{Acknowledgement}

This research has been supported by a Velux Stiftung Grant \#936 and a TDF grant \#186961-0611 from the Icelandic Centre of Research.

\section{References}

Ámundadóttir, M. (2016). Light-driven model for identifying indicators of non-visual health potential in the builtenvironment. Lausanne: EPFL.

Amundadottir, M., Rockcastle, S., Sarey Khanie, M., \& Andersen, M. (2017). A human-centric approach to assess daylight in buildings for non-visual health potential, visual interest and gaze behavior. Building \& Environment, 113, 521.

Correa, P., da Silva, V., \& Andersen, M. (2012). Influence of Shading control patterns on the energy assessment of office spaces. Energy \& Buildings.

DIVA-for-Rhino. Retrieved from http://diva4rhino.com/

Galasiu, A., \& Veitch, J. (2006). Occupant preferences and satisfaction with the luminous environment and control systems in daylit offices: a literature review. Energy and Buildings, 38, 728-742.

IES. (2014). Approved Method: IES Spatial Daylight Autonomy (SDA) and Annual Sunlight Exposure (ASE). Illuminating Engineering Society.

Jakubiec, A., \& Reinhart, C. (2011). The 'adaptive zone' - a concept for assessing glare through daylit spaces. Sydney: Proceedings to Building Simulation 2011.

Kleindienst, S., \& Andersen, M. (2012). Comprehensive annual daylight design through a goal-based approach. Building Research and Information, 154-173.

Kleindienst, S., Bodart, M., \& Andersen, M. (2008). Graphical representation of climate-based daylight performance to support architectural design. LEUKOS, 39-61.

Konis, K. (2016). A novel circadian daylight metric for building design and evaluation. Building and Environment.

Leslie, R.P., Radetsky, L.C., Smith, A.M. (2011). Conceptual Design Metrics for Daylighting. Lighting Research \& Technology.

Light Stanza. (n.d.). Retrieved from http://lightstanza.com/

Mardaljevic, J., \& Heschong, L. (2009). Daylight metrics and energy savings. Lighting Research and Technology, 41(3).

Moon, P., \& Spencer, D. (1942). Illumination for a non-uniform sky. Illuminating Engineering, 37(10), 797-826.

Rea, M., \& Figueiro, M. (2016). Light as a circadian stimulus for architectural lighting. Lighting Res. and Technol., 50(4).

Rockcastle, S., Amundadottir, M., \& Andersen, M. (2017). A Simulation-based workflow to assess human-centric daylight performance. Toronto: SimAUD 2017

Rockcastle, S., Amundadottir, M., \& Andersen, M. (2017). Contrast measures for predicting perceptual effects of daylight in architectural renderings. Lighting Research \& Technology, 49(7), 882-903.

Rockcastle, S., Amundadottir, M., \& Andersen, M. (2018). OCUVIS: A web-based visualizer for simulated daylight performance. Delft: SimAUD 2018.

Sefaira. Retrieved from https://sefaira.com/

Solemma. (2018). Alfa: Adaptive Lighting for Alertness A new circadian lighting design software. Retrieved from https://www.solemma.com/Alfa.html

Weinold, J. (2011). Dynamic daylight glare evaluation. Dynamic daylight glare evaluation (pp. 1250-1257). Sydney: Proceedings to IBPSA.

$W E L L v 2$. Retrived from https://v2.wellcertified.com/v/en/overview 